\title{
OCCHIO E VISIONE: MERAVIGLIA DELLA NATURA O PROGETTO DIFETTOSO?
}

\author{
PIER GIORGIO GOBBI (*) \\ Nota presentata dal m.e. F. Borsa \\ (Adunanza del 28 maggio 2015)
}

\begin{abstract}
SunTO. - La prestazione complessiva del sistema visivo umano presenta diversi difetti, tra i quali aberrazioni, monocromatiche e cromatiche, dinamica limitata, ridotta risoluzione spaziale e temporale. Perché si è evoluto in questo modo? Potrebbe essere migliorato in qualche aspetto, ad esempio con il supporto della tecnologia? La risposta è articolata sulla base di un modello neuro-fisico sviluppato dall'autore, in grado di fornire stime quantitative delle prestazioni ottiche e visive dell'occhio umano, in accordo con le misurazioni sperimentali. La conclusione, impostata sul principio di utilità, è che il progetto risultante dall'evoluzione naturale è perfettamente calibrato per le primarie esigenze di sopravvivenza dell'uomo primitivo in condizioni di illuminazione diurna e risulta difficilmente migliorabile dall'intervento umano.
\end{abstract}

$* * *$

ABSTRACT. - The behavior of the human visual system exhibits some flaws, including monochromatic and chromatic aberrations, finite dynamic range, limited spatial and temporal resolution. Why did it evolve this way? Can it be improved somehow, for example with the support of technology? The answer is obtained from a neuro-physical model developed by the author, which provides quantitative estimates of the optical and visual performances of the human eye, in agreement with experimental records. The conclusion, based on the principle of utility, is that the evolutionary design of the visual system is perfectly tailored for the primary survival needs of our primitive ancestors in daylight illumination, and it can hardly be improved with the help of human technology.

(*) IRCCS Ospedale San Raffaele, Milano, Italy.

E-mail: gobbi.piergiorgio@hsr.it 


\section{INTRODUZIONE}

Il titolo di questa memoria, che prospetta di sfiorare questioni inerenti ai «massimi sistemi», può evocare suggestioni di dispute filosofiche, magari fumose e inconcludenti. Niente di tutto ciò. Alla domanda del titolo cercherò di offrire una risposta il più possibile scientifica, fondata su dati quantitativi ed evidenze sperimentali; il fondamento è un modello psico-fisico - cioè congiuntamente ottico e neurologico - del meccanismo della visione umana.

Un'utile premessa discende da quanto riferiscono i neurofisiologi, per cui la visione è responsabile all'incirca del 90\% degli stimoli e delle informazioni che pervengono in ogni momento al nostro cervello. La percentuale sembra piuttosto elevata, ma può risultare ragionevole quando si considerino sia la quantità di informazione intrinsecamente legata ad un'immagine (si pensi ad es. alla differenza di dimensioni tra un file audio e un file video), sia il fatto che l'occhio lavora effettivamente «in continuo» - almeno in stato di veglia -, mentre gli altri organi di senso, pur essendo costantemente attivi, di fatto producono stimoli significativi soltanto al di sopra di una certa soglia o in presenza di variazioni importanti del segnale percepito. In corrispondenza a questo flusso imponente di informazione, si valuta che circa un terzo della massa cerebrale sia coinvolto nei processi visivi. Se così preponderante è l'importanza che la visione riveste per il nostro sistema neurologico, è legittimo interrogarsi sulla qualità delle informazioni che da essa provengono.

Se consideriamo il primo corno del dilemma, quello per cui l'occhio umano rappresenta una meraviglia della natura, esso origina a partire dal XVII sec., quando lo studio scientifico della visione inizia a mostrare la straordinaria complessità dei fenomeni fisici e biologici che vi sono implicati, studio ancora lungi dall'essere completato. Nel XIX sec. la questione inerente all'occhio è stata al centro della querelle tra creazionisti ed evoluzionisti. Ecco due testimonianze, entrambe concordi nel magnificare la "perfezione" del sistema visivo.

Nel 1802 il teologo creazionista William Paley in Natural Theology scrive:

there is to be seen, in every thing belonging to the eye and about it, an extraordinary degree of care

ed in esso vede "a miracle of design", alla stregua del meccanismo di un 
orologio. Mezzo secolo più tardi, Charles Darwin cita la perfezione e la complessità dell'occhio tra le difficoltà alla sua teoria dell'evoluzione naturale:

To suppose that the eye with all its inimitable contrivances for adjusting the focus to different distances, for admitting different amounts of light, and for the correction of spherical and chromatic aberration, could have been formed by natural selection, seems, I freely confess, absurd in the highest degree

(da On the Origin of Species, ch. VI, Organs of extreme perfection, 1859).

In seguito, Darwin supererà l'impasse sviluppando la teoria del gradualismo.

D'altra parte, è lo stesso progresso scientifico e tecnologico ad accendere i riflettori sui limiti dell'occhio: man mano che progredisce la comprensione delle leggi dell'ottica (geometrica e fisica) e aumenta la capacità tecnica di produrre ottiche in vetro di qualità sempre migliore, si appalesano vari difetti delle strutture rifrattive dell'occhio, in primis le aberrazioni. L'occhio umano soffre di aberrazione sferica (la risoluzione peggiora con l'aumentare del foro pupillare) e di altre distorsioni monocromatiche tra cui astigmatismo e coma; per di più le aberrazioni cromatiche (longitudinale e trasversale) distorcono l'immagine retinica a seconda del colore di un oggetto e del diametro pupillare (colori diversi vanno a fuoco su piani diversi, tipicamente il blu davanti alla retina, il rosso dietro). Difetti che già nell' 800 risultava relativamente semplice correggere ad es. in un obiettivo fotografico. In questi termini, il paragone tra l'ottica visiva e l'ottica strumentale risulta decisamente sfavorevole al "progetto occhio" elaborato dalla natura.

E' curioso notare a questo proposito che da secoli è invalso l'uso di spiegare ruolo e funzioni degli elementi costitutivi dell'occhio ricorrendo al paragone con una fotocamera: alla fine del '500 è G.B. Della Porta in Magia Naturalis il primo ad introdurre l'analogia tra l'occhio e una camera oscura con lente, ai tempi molto in voga per proiezioni pubbliche (anche se egli pone la sede dell'immagine nel cristallino). Oggi il paragone risulta ancora più efficace e completo con una videocamera digitale: in entrambi i casi si tratta di una "camera oscura" dove una lente proietta un'immagine capovolta del mondo esterno su una superficie sensibile alla luce. Si hanno così le sorprendenti equivalenze di Tab. 1. 
Tab. 1 - Corrispondenze tra "componenti" del sistema visivo e di una videocamera.

\begin{tabular}{ll}
\hline Occhio & Videocamera \\
\hline Cornea & Obiettivo fotografico (parte frontale) \\
\hline Pupilla & Diaframma \\
\hline Retina & Matrice CCD \\
\hline Coni / Bastoncelli & Pixels CCD \\
\hline Cristallino (variazione di forma) & Autofocus (traslazione di lenti) \\
\hline Nervo ottico & Cavo di segnale \\
\hline Corteccia cerebrale & Scheda di memoria e software di elaborazione \\
\hline
\end{tabular}

In Tab. 2 sono riportati alcuni dei parametri tipici dell'occhio umano per avere elementi di raffronto con gli analoghi parametri di strumenti tecnologici. E' interessante come alcuni di essi siano assolutamente confrontabili, mentre altri decisamente discordanti (l'occhio è molto sensibile, capace di rilevare 5-9 fotoni entro $0.1 \mathrm{~s}$, ma è pure molto lento; di rilievo il numero di fotorecettori in raffronto con i pixels di videocamere commerciali; la retina di forma sferica consente un amplissimo campo visivo).

\begin{tabular}{ll}
\hline \multicolumn{1}{c}{ Tab. 2. Valori tipici di alcuni parametri dell'occhio umano. } \\
\hline Lunghezza focale occhio & $\mathrm{f}=17 \mathrm{~mm}$ \\
\hline Diametro pupilla & $\mathrm{da} 2 \mathrm{a} 8 \mathrm{~mm}$ \\
\hline Apertura numerica & $\mathrm{da} \mathrm{f} / 2.1 \mathrm{a} \mathrm{f} / 8.5$ \\
\hline Autofocus (a 20 anni) & $\mathrm{da} 10 \mathrm{~cm} \mathrm{a} \infty$ \\
\hline Diametro retina & $\mathrm{D}=32 \mathrm{~mm}$ \\
\hline Numero fotorecettori & $130 \mathrm{milioni}$ \\
\hline Numero fotorecettori a colori & $6 \mathrm{milioni}$ \\
\hline Sensibilità ISO & $\mathrm{da} 0.25 \mathrm{a} 60.000 \mathrm{ca}$ \\
\hline Tempo di risposta & $0.1 \mathrm{~s}$ \\
\hline Campo visivo & $170^{\circ} \times 150^{\circ} \mathrm{ca}$ \\
\hline
\end{tabular}

Oltre a tali parametri andrebbero considerate le capacità di pulizia e lubrificazione autonome (film lacrimale), la possibilità di autoriparazione (epitelio corneale), la vita media di 80 anni (pur con progressivo degrado), l'alimentazione energetica molto efficiente, ecc. Tuttavia la tabella non aiuta a pervenire ad una valutazione assoluta delle proprietà dell'occhio. Come accennato, la mancata correzione delle aberrazioni costituisce un indubbio limite prestazionale. In un recente lavoro dedi- 
cato a una revisione critica del progetto ottico dell'occhio (1), lo spagnolo Navarro arriva a sostenere, severamente ma non senza qualche ragione, che

the eye presents a poor optical quality on axis..., is highly aberrated except for small pupils $(<3 \mathrm{~mm})$, young subjects, on-axis and under monochromatic illumination.

Praticamente, un mezzo disastro. Ma perché è così, se lo studio anatomico e funzionale dell'occhio rivela oggi un quadro complessivo di architettura e soluzioni adottate del tutto stupefacenti, inimmaginabili due secoli fa da Paley e Darwin?

Una prima giustificazione può essere prodotta in modo immediato sulla base di semplici considerazioni pratiche. Se si considerano le due aberrazioni che affliggono primariamente la prestazione visiva aberrazione sferica e aberrazione cromatica longitudinale -, va notato che nella pratica tecnologica è possibile conseguirne l'annullamento quasi totale con l'impiego di doppietti, cioè di coppie di lenti, cementate tra loro o spaziate in aria, costituite di vetri con indici di rifrazione molto diversi, denominati crown (basso indice, tipicamente 1.55) e flint (alto indice). Con un opportuno progetto delle due lenti, si riesce ad ottenere che l'aberrazione sferica di un componente sia circa uguale ma di segno opposto a quella dell'altro, così da elidersi a vicenda, e così pure che le aberrazioni cromatiche longitudinali dei due elementi si compensino efficacemente su un ampio intervallo di lunghezze d'onda. Nel caso dell'occhio la possibilità è compromessa dall'uso, per i suoi elementi rifrattivi (cornea e cristallino), di tessuti biologici costituiti prevalentemente da polimeri organici ad alto contenuto di acqua (collagene di vari tipi); in questi materiali l'indice di rifrazione risulta molto simile a quello dell'acqua (1.333) ed il ventaglio di variabilità ottenibile mediante inclusione di altre molecole organiche è molto ridotto (tipicamente da 1.336 a 1.406).

Se ciò rappresenta un ostacolo insormontabile per quanto concerne l'aberrazione cromatica, così non è per la sferica, in quanto esistono altre modalità per correggere l'aberrazione sferica anche in elementi ottici singoli. Una possibilità è di progettare lenti con superfici che siano calotte non di una sfera bensì di un ellissoide (come un pallone da rugby), realizzando così lenti asferiche. Un'altra possibilità è di progettare lenti aventi indice di rifrazione non uniforme, ma con una variazio- 
ne continua nel volume delle stesse, ottenendo cioè le cosiddette ottiche GRIN (GRaded INdex). Ebbene entrambe le modalità sono riscontrabili negli elementi rifrattivi dell'occhio. La superficie anteriore della cornea, che è responsabile dell' $83 \%$ del potere rifrattivo totale dell'occhio, ha una superficie asferica di tipo prolata (più curva in asse che in periferia, come il pallone da rugby su una calotta lungo l'asse maggiore). Per contro il cristallino, oltre ad avere superfici pure prolate, esibisce una marcata variazione di indice sia assialmente (basso-alto-basso) che radialmente (decrescente dall'asse alla periferia). Entrambe le circostanze cooperano nella direzione di ridurre l'aberrazione sferica complessiva dell'occhio, rispetto al caso di cornea sferica e cristallino uniforme, ma non abbastanza da annullarla; una componente significativa permane, come sperimentabile da chiunque nella ridotta acuità visiva con grandi diametri pupillari (es: difficoltà di lettura con illuminazione fioca).

Per dirla con Navarro:

the optical system of the eye seems to combine smart design principles with outstanding flaws... which contribute to deteriorate the final optical quality... Somehow, there could have been an opportunity (in the evolution) to have much better quality, but this was irreparably lost.

Ecco dunque ridefinita la questione posta nel titolo: davvero l'evoluzione avrebbe potuto scegliere un progetto migliore per l'ottica visiva? E se sì, quale sarebbe stato il vantaggio visivo e per quali plausibili motivi non è stato realizzato? Lo stesso autore citato, in un altro articolo (2), fornisce le linee guida di metodo per trovare una risposta:

However, the brain, and not the eye, is the organ that is responsible for vision... Consequently, vision cannot be understood in terms of optics alone; on the contrary, optics serves to the neural system. Then, any explanation should necessarily consider the main neural process.

Una considerazione peraltro supportata dall'evidenza che embriologicamente l'occhio deriva da una differenziazione del sistema nervoso in fase di sviluppo.

La strada da percorrere per trovare risposta alla domanda iniziale si fa più complessa: non basta studiare la proiezione dell'immagine sulla retina e caratterizzarne le proprietà in rapporto all'oggetto che quell'immagine ha creato, occorre estendere l'analisi all'immagine percepita 
dalla mente umana, includendo quindi il processo neuronale di interpretazione dell'immagine proiettata. E' l'ambito della psico-fisica che, a dispetto della sensazione di vaghezza che il nome può suscitare, è una disciplina scientifica rigorosa, volta a determinare relazioni quantitative tra una percezione soggettiva umana e lo stimolo esterno che l'ha generata. L'ipotesi di base è che il sistema percettivo umano sia assimilabile a uno strumento di misura che dà risultati (sotto forma di risposte e/o giudizi) che possono essere analizzati in modo sistematico. Ma come valutare - anche quantitativamente - questa immagine percepita, che non è accessibile all'esterno? Non c'è altra soluzione che farsela descrivere dal soggetto stesso che la percepisce.

E' quanto avviene correntemente nella diffusissima pratica di misurazione del visus di un soggetto, cioè di quantificazione della sua capacità visiva. La descrizione dell'immagine percepita è relativa a oggetti standard, scelti opportunamente, quali ad esempio i caratteri di una tavola optometrica. In questo caso la grandezza psico-fisica che viene misurata è l'acuità visiva $(\mathrm{AV})$, cioè la capacità di risolvere spazialmente dettagli sempre più piccoli di un oggetto. La misura della AV è espressa in frazioni adimensionali (decimi in Europa, ventesimi in USA, sesti in UK), dove il valore di riferimento " 1 " (cioè 10 decimi) è relativo ad un angolo sotteso di 1 minuto d'arco (1/60 di grado, pari a ca. 0.3 $\mathrm{mrad})$. Avere una AV di 10/10 equivale perciò a riuscire a risolvere dettagli con dimensione angolare di 1'; un valore di AV minore di 1 indica un visus inferiore, ad es. 5/10 corrisponde a una risoluzione di 2' e viceversa. Le «linee» di un ottotipo hanno caratteri di dimensione decrescente, in modo che ogni 3 linee la dimensione dimezzi, e dopo 10 linee sia ridotta a $1 / 10$. La progressione delle linee è dunque logaritmica, non lineare (come logaritmiche sono tutte le risposte sensoriali: legge di Weber-Fechner); il rapporto dimensionale tra una linea e la successiva è costante $(\sim 1.25)$. Così facendo, si è considerata la grandezza psicofisica $(\mathrm{AV})$ come funzione misurabile dello stimolo esterno (dimensione del carattere).

A questo punto è opportuno precisare la differenza tra visus e refrazione, due categorie d'uso corrente collegate tra loro ma spesso equivocate l'una con l'altra. Per refrazione (curiosamente, nel linguaggio medico è invalso l'uso del termine refrazione, mentre in ottica si usa rifrazione, pur condividendo entrambi la stessa radice semantica) si intende la procedura con cui si valuta il difetto ottico, ovvero si determina il potere della lente correttiva che produce il miglior risultato visi- 
vo. L'emmetropia è la condizione in cui l'occhio vede al meglio delle proprie possibilità, senza bisogno di alcuna correzione. In tal caso l'immagine di un oggetto all'infinito (una stella) viene proiettata dall'ottica oculare (cornea e cristallino) esattamente sulla retina del soggetto. I difetti ottici di tipo sferico sono:

- miopia: è la condizione in cui l'immagine si forma davanti alla retina, vuoi per un'abnorme lunghezza del bulbo oculare, o per una cornea particolarmente curva. In tal caso per posizionare l'immagine sulla retina occorre utilizzare una lente correttiva divergente, caratterizzata da un potere diottrico negativo (lenti da $-1,-2,-5 \mathrm{D}$, ecc.);

- ipermetropia: è la condizione duale alla miopia: l'immagine si forma dietro la retina e la correzione impiega una lente correttiva convergente con potere diottrico positivo (lenti da $+1,+2 \mathrm{D}$, ecc.).

Dunque la refrazione, misurata in diottrie, attiene all'immagine retinica proiettata mentre il visus, misurato in decimi di AV, valuta l'immagine percepita. La refrazione valuta solo l'ottica oculare, il visus l'intero sistema visivo. La relazione esistente tra le due quantità è estremamente complessa e dipende da una quantità di fattori di natura ottica, strutturale e neurofisica. Per di più la presenza di lesioni o patologie delle strutture neuro-oftalmiche può precludere ogni possibilità di inferenza da refrazione a visus: è chiaro infatti che l'emmetropia è condizione necessaria per avere un buon visus naturale (senza correzione con lenti!) ma non è condizione sufficiente, in quanto danni alla retina o al nervo ottico possono ridurre drasticamente il visus fino alla cecità.

\section{METODI}

Uno strumento teorico capace, tra le altre cose, di quantificare la relazione esistente tra refrazione e visus di un soggetto neurologicamente normale, per varie situazioni di difetto visivo, è fornito dal modello CAGE-Barten, che ho sviluppato qualche anno fa (3). Più in generale il modello è in grado di quantificare le proprietà ottiche e percettive del sistema visivo umano. Come ovvio dalla digressione precedente, si tratta di un modello misto in quanto combinazione di un modello ottico di occhio (CAGE) che riproduce l'immagine retinica proiettata e di un modello neuro-fisico (Barten) che riproduce l'immagine percepita, simulando la trasduzione dello stimolo ottico in segnale elettrico (e biochimico) e la successiva elaborazione del segnale operata dalle strutture 
neuronali (retina, nervo ottico, corteccia cerebrale). Mi limito ad illustrare brevemente le caratteristiche salienti del modello CAGE-Barten, giusto per agevolare la discussione dei risultati.

Modello ottico (CAGE): in letteratura si ha una vasta disponibilità di modelli ottici dell'occhio umano, con livelli di complessità e di accuratezza molto vari. Io mi sono avvalso di un modello storico ampiamente diffuso, il cosiddetto schema "esatto" di Gullstrand (4), un oculista svedese che vinse il premio Nobel per la fisiologia/medicina nel 1911 per i suoi "studi sui mezzi diottrici dell'occhio". Il modello di Gullstrand consta di 6 superfici, 2 per la cornea e 4 per il cristallino, con una zona centrale a indice più alto, per simulare il gradiente d'indice che si rileva nell'occhio umano. Per poter utilizzare il modello di Gullstrand per la riproduzione di immagini retiniche, occorre aggiungere alcune caratteristiche che ne estendano il campo di applicazione senza alterare le proprietà originarie. Tra queste, l'introduzione di superfici asferiche (tutte le interfacce dell'occhio umano lo sono) e l'introduzione della dispersione cromatica (il modello originario è monocromatico). I parametri asferici delle superfici sono stati ottenuti attraverso una complessa procedura di replicazione di immagini retiniche sperimentali (5). Un'ulteriore validazione del modello ottico è giunta dal confronto delle sue predizioni di aberrazione sferica con dati sperimentali. Il modello "potenziato" risultante è stato chiamato CAGE (Chromatic Aspherical Gullstrand Exact).

Modello psico-fisico (Barten): il nucleo del modello è derivato da quello sviluppato dall'olandese Barten negli anni '90 (6), basato sulla semplice assunzione che la risposta del sistemo visivo è quantitativamente determinata dal livello di rumore presente in esso. Il rumore, cioè la fluttuazione casuale del segnale visivo attorno al suo valor medio, è dovuto sia al flusso di fotoni incidente sulla retina (photon shot noise) che alle fotocorrenti lungo il nervo ottico, in molteplici percorsi paralleli dalla retina alla corteccia visiva. L'effetto del rumore è di stabilire una soglia sotto la quale un'immagine non può essere percepita senza un'alta probabilità d'errore (un effetto paragonabile al disturbo portato dalla nebbia all'aperto o dal flicker nell'immagine TV analogica). Il modello di Barten è concettualmente abbastanza semplice, ma la dipendenza da molte variabili (tra le quali: lo spettro dell'illuminazione, il livello di illuminanza retinica, le dimensioni spaziale e temporale dell'oggetto osservato) ne complica alquanto l'espressione analitica. Anche in questo caso il modello è stato opportunamente modificato per 
risolverne alcune inconsistenze interne ed ampliarne le possibilità applicative.

I parametri numerici del modello di Barten modificato (una decina) sono stati ricavati da una procedura di best fitting di molte centinaia di dati psico-fisici tratti dalla letteratura e relativi a misura di curve di sensibilità al contrasto (CSF, Contrast Sensitiviy Function) in decine di soggetti. La rilevazione della curva CSF si basa sulla osservazione di barre sinusoidali di luminosità (Fig. 1) al posto di caratteri alfanumerici come nel caso di misura di AV. Tali barre sono variate in contrasto finché risultano non più percepibili dal soggetto sotto test, e la misura ripetuta variando la frequenza spaziale (spaziature tra le barre). In Fig. 2 risulta evidente come la visibilità delle barre diminuisca col diminuire del contrasto e con l'aumentare del livello di rumore, fino ad essere preclusa del tutto. La curva CSF così ottenuta fornisce molte più informazioni della semplice misura di AV, la quale rappresenta soltanto un punto della curva CSF; inoltre l'impiego di barre sinusoidali elimina l'effetto "congettura" connesso all'uso di caratteri con un proprio livello di riconoscibilità, consentendo una misura più oggettiva. Per contro, la rilevazione della CSF risulta una procedura molto lunga e impegnativa, e solo raramente è effettuata nel corso di esami optometrici ordinari.
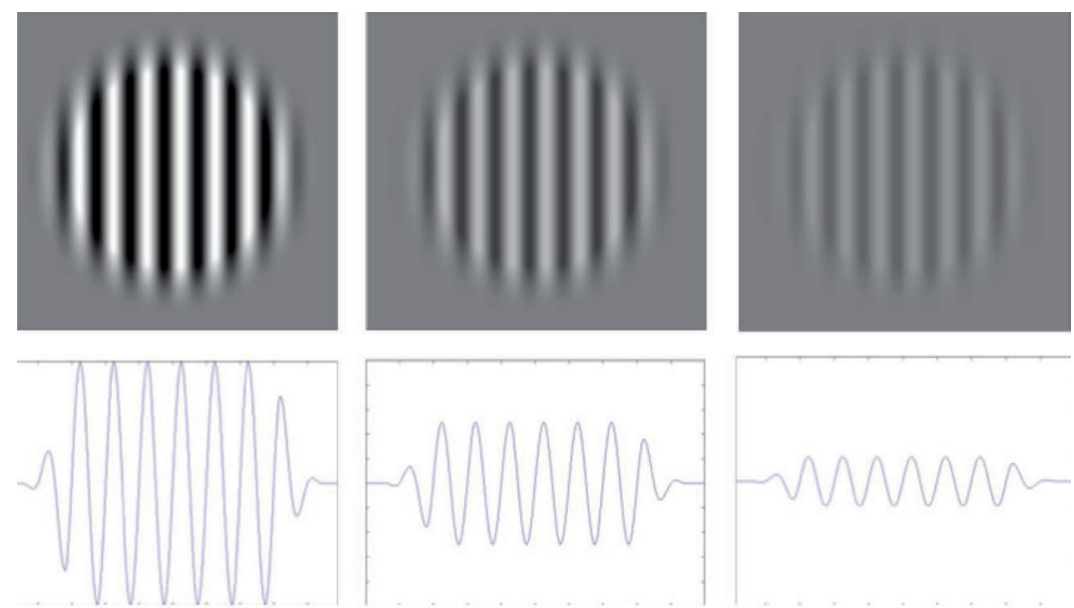

Fig. 1 - Barre sinusoidali di luminanza, a contrasto variabile. Il contrasto è definito come la variazione massima di luminanza (max - min) in rapporto alla somma delle luminanze massima e minima (max + min).

Nei tre esempi soprastanti il contrasto vale 100\% (sinistra), 50\% (centro) e 20\% (destra). 

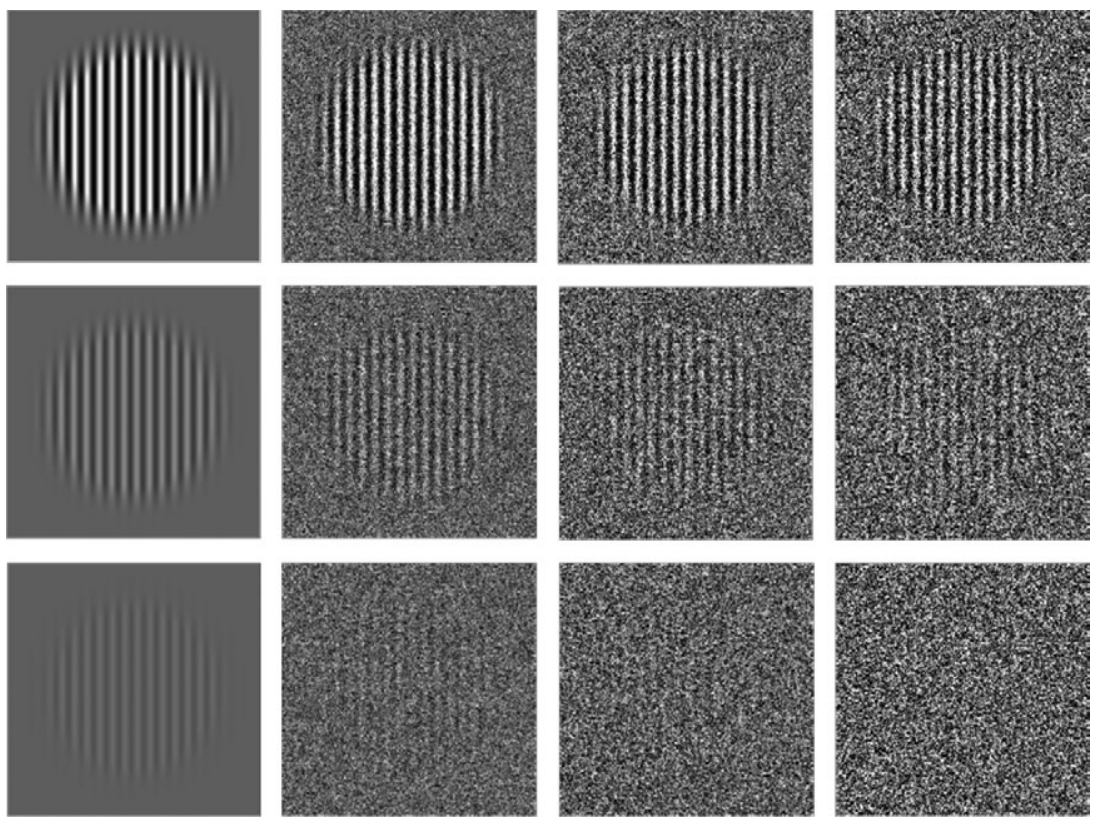

Fig. 2 - Barre sinusoidali di luminanza mostrate per diversi valori di contrasto (costante in ogni riga; dall'alto: 100\%; 30\% e 10\%) e diversi livelli di rumore (costante in ogni colonna; da sinistra: 0; 20\%; 33\%; 50\%).

Il livello di rumore è definito come il rapporto tra il valore quadratico medio (RMS) del rumore e il valore medio del segnale di luminanza. Il rumore è discretizzato spazialmente ("pixelato") per rispettare la discretizzazione dei fotorecettori retinici.

In conclusione, il modello ottico può calcolare con accuratezza l'immagine retinica proiettata in un occhio qualsivoglia; tramite la CSF, calcolata dal modello psico-fisico per quello stesso occhio con pari accuratezza, è possibile ricostruire l'immagine percepita, cioè l'informazione visiva disponibile alla mente, dopo il processo di acquisizione ed elaborazione. Va sottolineato da ultimo che il modello CAGE-Barten è un modello puramente fisico, basato solo su una descrizione fisica dei processi neuro-ottici che hanno luogo nella visione; inoltre è deterministico e non basato su assunzioni euristiche sviluppate ad hoc. 


\section{RisUltaTI}

La Fig. 3 mostra, a titolo dimostrativo, uno dei risultati ottenibili con il modello testé descritto: si tratta del confronto tra i valori di AV misurati sperimentalmente (barre) in una popolazione emmetrope (7), suddivisi per fasce d'età, e la predizione fornita dal modello nelle stesse condizioni ambientali della misura (linea continua). Pur trattandosi di dati non perfettamente omogenei (i dati si riferiscono ad ottotipi alfabetici, la predizione teorica è desunta dalla curva CSF), l'accordo è eccellente: la previsione del modello coincide con i valori riscontrati nella fascia d'età 18-40 anni, ovvero risulta superiore di neppure mezza linea di AV al valor medio tra 18 e 80 anni (linea tratteggiata). Per inciso il valore di AV si attesta intorno a 14/10.

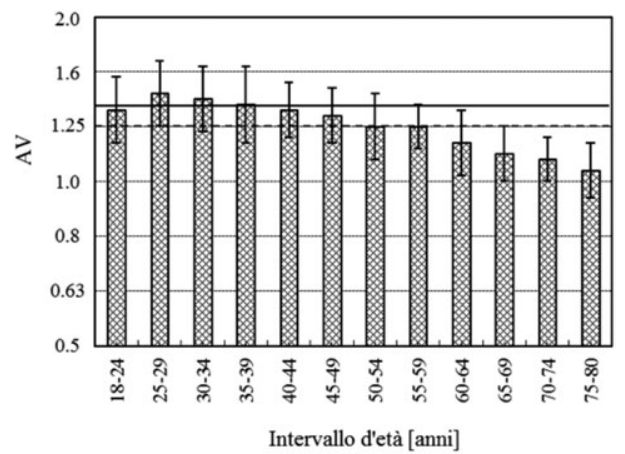

Fig. 3 - Andamento dell'acuità visiva in funzione dell'età anagrafica.

Barre: dati sperimentali da Elliott et al. (7); linea tratteggiata: valor medio da 18 a 80 anni. Linea continua: previsione del modello CAGE-Barten.

Dati e simulazione di Fig. 3 si riferiscono a una luminanza (luminosità dell'ottotipo) di $160 \mathrm{~cd} / \mathrm{m}^{2}$. La risposta della pupilla alla luminosità ambientale (riflesso pupillare) varia sensibilmente da individuo a individuo, sia pur entro l'intervallo obbligato di Tab. 2 e dipende da pigmentazione dell'iride, errore refrattivo, condizioni emotive, ecc.; il modello assume un andamento medio del riflesso pupillare misurato nella popolazione. Se si graficano i valori di AV per ogni valore di luminanza ambientale, si ottiene la curva inferiore (A) di Fig. 4. L'asse orizzontale inferiore riporta i valori di luminanza (con scala logaritmica) mentre quello superiore indica i valori corrispondenti di diametro 
pupillare medio. Per facilitare la lettura della Fig. 4, in Tab. 3 sono riportati dei valori indicativi di luminanza relativi ad alcune situazioni ambientali comuni.

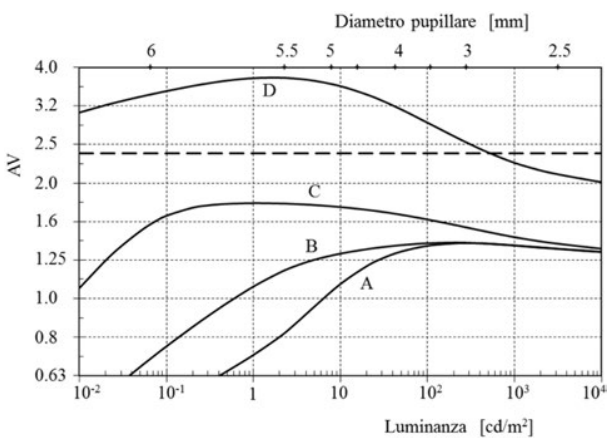

Fig. 4 - Grafico dell' acuità visiva in funzione della luminanza ambientale; lungo l'asse orizzontale superiore sono riportate le dimensioni del foro pupillare corrispondente ad ogni luminanza (valor medio). Tutte le curve sono calcolate per mezzo

del modello CAGE-Barten. Curva A: occhio reale. Curva B: derivata dalla curva A senza aberrazione sferica. Curva $C$ : derivata dalla $B$ senza aberrazione cromatica.

Curva D: derivata dalla $C$ senza elaborazione neuronale dell' informazione visiva. Retta tratteggiata: limite massimo di acuità visiva fissato dalla densità dei coni in fovea (soglia di aliasing).

Tab. 3. Valori di luminanza corrispondenti ad alcune condizioni di illuminazione.

\begin{tabular}{ll}
\hline Ambiente & Luminanza $\left[\mathbf{c d} / \mathbf{m}^{2}\right]$ \\
\hline Nevaio assolato & 10.000 \\
\hline Cielo sereno con atmosfera tersa & 10.000 \\
\hline Luna piena & 3.000 \\
\hline Cielo nuvoloso & 1.000 \\
\hline Cielo nuvoloso cupo & 100 \\
\hline Interno di giorno & 100 \\
\hline Esterno di notte & 0,01 \\
\hline Visione fotopica (solo coni) & $>3$ \\
\hline Visione mesopica (coni + bastoncelli) & $0,001-3$ \\
\hline Visione scotopica (solo bastoncelli) & $<0,001$ \\
\hline
\end{tabular}

La curva A di Fig. 4 mostra che l'acuità visiva è massima attorno a $300 \mathrm{~cd} / \mathrm{m}^{2}$ e declina rapidamente col diminuire della luminanza, rimanendo superiore a $8 / 10$ in tutto l'intervallo della visione fotopica. Il 
lieve declino alle luminanze più elevate è da attribuire al fenomeno ottico della diffrazione, in corrispondenza a dimensioni pupillari molto piccole. (E' a causa della diffrazione che una lente non può concentrare un fascio di luce in un punto di dimensione zero, come pure un fascio di luce non può propagarsi perfettamente collimato, ma diverge in modo più o meno accentuato).

Una peculiarità del modello CAGE-Barten risiede nella possibilità di valutare gli effetti sulla visione di vari fattori limitanti, tra quelli citati. Ad esempio, si può modificare o addirittura annullare un'aberrazione, indipendentemente da ogni altro parametro, e calcolare la variazione risultante della prestazione visiva; è come disporre di una scatola nera provvista di manopole con cui poter variare i parametri progettuali dell'occhio, in una sorta di sfida virtuale all'evoluzione per fare di meglio! Gli unici effetti che non possono essere eliminati sono quelli legati a leggi fisiche fondamentali della natura: la diffrazione e il rumore.

La curva B di Fig. 4 si riferisce al caso di un occhio totalmente privo di aberrazione sferica, un risultato in linea di principio alla portata della chirurgia fotorefrattiva corneale. Per luminanze maggiori di 100 $\mathrm{cd} / \mathrm{m}^{2}$ le curve A e B sono indistinguibili, in conseguenza del fatto che per piccoli diametri pupillari l'aberrazione sferica dell'occhio è trascurabile. Le due curve si discostano al di sotto di $100 \mathrm{~cd} / \mathrm{m}^{2}$, con un vantaggio di prestazione che cresce col diminuire della luminanza, fino a due linee di AV (vale a dire due righe di un ottotipo alfabetico) in condizioni di penombra $\left(1 \mathrm{~cd} / \mathrm{m}^{2}\right)$. La curva $\mathrm{B}$ di fatto preclude ogni possibilità di migliorare la massima acuità visiva con un rimodellamento della superficie corneale.

Il secondo passo è naturalmente di azzerare anche l'aberrazione cromatica longitudinale, in aggiunta all'aberrazione sferica, un traguardo peraltro quasi irrealizzabile su un occhio reale mediante correzione ottica (lenti), se non per un solo angolo di vista. Il risultato è rappresentato dalla curva $\mathrm{C}$ di Fig. 4. In queste condizioni il miglioramento è più marcato e risulta apprezzabile già attorno a $1000 \mathrm{~cd} / \mathrm{m}^{2}$; per di più l'AV raggiunge un massimo, più alto, di circa $18 / 10$ a $1 \mathrm{~cd} / \mathrm{m}^{2}$.

Il terzo e ultimo passo comporta un intervento non sull'ottica dell'occhio ma sull'elaborazione del segnale visivo svolta dalle varie strutture neuronali; si tratta di escludere ogni tipo di elaborazione parallela e in tempo reale dell'immagine retinica e di riferirsi semplicemente ai dati grezzi acquisiti dai fotorecettori. Rimuovendo dal modello questa attività neuronale, si ottiene la curva D di Fig. 4. In tal 
caso l'aumento di prestazione visiva è strabiliante: il massimo di $\mathrm{AV}$ sfiora i 40/10 in condizioni mesopiche $\left(2 \mathrm{~cd} / \mathrm{m}^{2}\right)$, e anche a luminanze elevate rimane sopra i 20/10, con un vantaggio teorico tra 2 e 7 linee di AV. Va subito detto che una tale prestazione visiva sarebbe solo virtuale: infatti la densità di coni nella parte centrale della retina limita necessariamente la AV a non superare i 24/10 (v. curva tratteggiata di Fig. 4). Ogni punto al di sopra di tale limite incorre nel cosiddetto fenomeno di "aliasing", un artefatto per cui qualche cosa di falso viene percepito in conseguenza del fatto che il segnale risulta discretizzato con meno risoluzione di quanto imposto dal teorema del campionamento (l'esempio equivalente più comune, nel dominio temporale, è l'effetto "ruote del carro", che, in una visualizzazione TV o cinematografica, possono essere viste ruotare in verso opposto al normale senso di avanzamento). Per supportare un'effettiva AV di 40/10 sarebbe necessaria una densità di coni tripla.

\section{Discussione}

Il confronto tra le curve A e D di Fig. 4, vale a dire tra prestazione visiva reale e potenziale, sembra indicare un difetto piuttosto grossolano del sistema visivo umano così come prodotto dall'evoluzione naturale. Infatti il sacrificio di svariate linee di AV sembra rappresentare un errore di progettazione, per la presenza non solo di aberrazioni non corrette (almeno non completamente), ma anche di scelte funzionali opinabili, quale il processamento parallelo e in tempo reale dell'informazione visiva.

Ma... è proprio qui che risiede lo snodo della questione.

La visione è un processo straordinariamente complesso, al di là di quanto ci si possa rendere conto. Abituati come si è ad una valutazione funzionale della propria prestazione visiva fondata in buona misura sulla misura dell'AV e poco più (campo visivo, test cromatico, sensibilità al contrasto), si tende ad enfatizzare l'importanza dell'AV, da vicino e da lontano, come indicatore principe della "bontà" dei propri occhi. Dal punto di vista operativo è certamente così: l'AV deve servire da parametro di riferimento assoluto per la correzione con lenti dei difetti di refrazione e la conseguente valutazione del visus ottenibile. E' abbastanza chiaro che un deficit significativo dal livello "normale" di 10/10 presuppone la presenza di qualche anoma- 
lia oculare. Diverso è il caso quando si discute, come qui, di valutazione teorica del sistema visivo.

In questo contesto più ampio, va detto che l'AV è semplicemente uno dei tanti aspetti della visione, non l'unico e forse nemmeno il più importante di tutti. Fondamentalmente, il sistema visivo umano fornisce al cervello immagini del mondo circostante ad un ritmo di diversi "quadri" al secondo (in analogia con l'immagine TV o cinematografica), un ritmo che si accorda con i tempi di risposta tipici dell'apparato muscolo-scheletrico dell'uomo. Per un'interazione efficace con l'ambiente, tale informazione visiva deve essere analizzata in modo approfondito e immediato: per prendere decisioni tempestive, come fuggire da un pericolo, non c'è possibilità di post-elaborazione.

A questo scopo il sistema visivo scompone l'immagine proiettata sulla retina in un gran numero di frammenti di informazione a basso livello, che sono inviati ad aree diverse della corteccia visiva, ciascuna specializzata ad estrarre specifici aspetti elementari dall'immagine osservata. Tra questi: orientazione, dimensione, contorno, movimento, velocità, distanza, profondità, trama superficiale, colore, contrasto, ecc.; per ciascuna di queste caratteristiche il sistema visivo è poi particolarmente efficace nel rilevare variazioni temporali piuttosto che condizioni stazionarie, e quindi l'attenzione è maggiormente attirata dai cambiamenti di orientazione, dimensione, velocità, ecc. Il meccanismo con cui l'immagine retinica è frazionata e convogliata in una miriade di canali paralleli è la somma/sottrazione di informazioni provenienti da diverse porzioni dell'immagine e il successivo confronto degli output. Elementi base di questa scomposizione sono i neuroni del sistema visivo, in cui il segnale di uscita dipende dalla somma algebrica dei segnali in ingresso: quelli eccitatori col segno positivo, quelli inibitori col segno negativo, con coefficienti di peso assegnati ai diversi ingressi. Una forma tipica della distribuzione dei segnali afferenti a un neurone contempla una zona centrale eccitatoria e una periferia inibitoria, ma è presente anche la versione duale; la simmetria può essere circolare o elongata lineare; la dimensione spaziale del dominio degli ingressi (campo ricettivo) è estremamente variabile da neurone a neurone (8). Questa architettura di suddivisione e ricombinazione del segnale è ripetuta in tutti gli strati neuronali, lungo l'intero percorso visivo dalla retina fino alla corteccia, con diverse forme, dimensioni e polarità dei campi ricettivi coinvolti.

Il processo sottrattivo descritto, denominato inibizione laterale, 
produce una quantità di effetti; il più semplice forse da comprendere è l'esaltazione dei contorni spaziali che esso comporta, quale risultato della maggiore amplificazione del segnale in corrispondenza a discontinuità di luminanza rispetto a punti di luminanza costante (equivalente ad un derivatore spaziale, o ad un filtro passa-alto). Le conseguenze di questa architettura funzionale sono varie: la distorsione dell'immagine percepita rispetto all'immagine proiettata sulla retina; il filtraggio delle frequenze spaziali contenute nell'immagine con una riduzione della risoluzione spaziale (minore AV); l'apporto di rumore elettrico per il trasporto del segnale lungo molteplici canali in parallelo, a causa di fluttuazioni casuali in ciascun canale.

L'esistenza stessa di aree di corteccia visiva specializzate nell'estrazione di specifiche informazioni dall'immagine retinica dimostra che il sistema visivo umano si è evoluto per esaminare, analizzare e predire le visualizzazioni più rilevanti e ricorrenti nella vita di ogni giorno, facilitando compiti decisivi per la sopravvivenza come riconoscere gli amici dai nemici, scoprire i frutti maturi, riconoscere i predatori e le prede, valutare distanza e velocità dei bersagli, prevedere la traiettoria di oggetti e così via. L'analisi estremamente complessa svolta dalla rete neuronale visiva si traduce in abilità straordinarie e spesso sottovalutate, una per tutte la capacità di riconoscere i volti molto rapidamente e da solo pochi dettagli, nonché di decodificare le espressioni e perfino gli umori da qualche impercettibile traccia su un volto. Così è dimostrato che esistono aree neuronali specifiche dedicate alla codifica dei volti, delle loro identità ed espressioni (9); al punto che lesioni in tali aree possono portare all'incapacità di riconoscere volti familiari e/o le loro espressioni (prosopoagnosia). Queste medesime capacità sono anche alla base delle illusioni ottiche, che si verificano ogniqualvolta il sistema visivo è stimolato da scene innaturali e da contesti ingannevoli.

Non è possibile quantificare il guadagno in prestazione visiva derivante dallo sviluppo di tutte queste abilità, sì da contrapporlo alla corrispondente perdita di AV testimoniata dalla Fig. 4. Si può soltanto paragonare la procedura neuronale di tipo parallelo con una procedura seriale che preservi la massima risoluzione dell'immagine acquisita, ad esempio per il riconoscimento di un volto. In questo caso, in analogia con quanto avviene nell'elaborazione informatica, la sequenza di passi sarebbe: 1: immagine proiettata. 2: immagine acquisita ad alta risoluzione. 3: estrazione dei parametri identificativi significativi. 4: confronto 
dei parametri individuati con il data base dei volti noti. 5: riconoscimento del volto osservato. Nel caso del sistema visivo, i passi 2-3-4 sono svolti simultaneamente lavorando su un'immagine acquisita a risoluzione inferiore, con evidente risparmio di tempo.

E' possibile invece quantificare, sia pure grossolanamente, il vantaggio derivante dall'adozione di una visione tricromatica, pur con penalizzazione in $\mathrm{AV}$, rispetto a un'opzione puramente monocromatica. Con riferimento di nuovo alla Fig. 4, si è detto che la curva $\mathrm{C}$ rappresenta la prestazione in $\mathrm{AV}$ di un occhio corretto per aberrazione cromatica longitudinale (oltre che per aberrazione sferica). Di fatto, la stessa curva può essere vista come descrittiva della prestazione di un occhio umano virtuale avente un solo fotopigmento al posto dei tre spettralmente distinti che contraddistinguono l'occhio umano reale. Cioè un occhio monocromatico, in cui la rappresentazione del mondo esterno sarebbe solo in gradazioni di grigio. Un sistema visivo molto simile a quello di molti animali, infatti la curva $\mathrm{C}$ di Fig. 4, con il picco di AV in condizioni di forte penombra, sembra tipico di predatori notturni quali felini e rapaci. Ora stanti le abitudini prettamente diurne dell'uomo cacciatore-raccoglitore - e di tutti i primati prima di lui - la scelta evolutiva è stata di privilegiare le prestazioni fotopiche del sistema visivo a scapito di una perdita di una-due linee di AV in condizioni mesopiche. Il vantaggio di una visione a colori è dato dalla capacità di discriminare qualche milione di colori differenti a fronte di qualche centinaio di livelli di grigio per una visione monocromatica. Questo guadagno (naturalmente al costo anche di una rete neuronale più grande per gestire l'informazione cromatica) indica che la Fig. 4 è sostanzialmente ingannevole: descrive infatti solo una prestazione di luminanza, dato che i test optometrici, caratteri o barre sinusoidali, sono monocromatici (bianco/nero/grigio). In realtà una metrica opportuna della prestazione visiva a colori richiede altri due parametri, tinta e saturazione, oltre alla luminanza; di conseguenza, la Fig. 4 rappresenta solo la proiezione sul piano della luminanza di un grafico intrinsicamente quadridimensionale ( $A V$ vs. luminanza, tinta, saturazione). E il numero di dimensioni aumenta vertiginosamente quando si considerino tutti i parametri (dimensione, velocità, ecc.) a cui il sistema visivo applica la propria elaborazione.

Ritornando alla curva A di Fig. 4 (comportamento di un occhio reale), la presunta "modesta" prestazione va riconsiderata alla luce delle opzioni evolutive di visione cromatica ed elaborazione parallela 
dell'informazione visiva. In questa prospettiva emerge con chiarezza che l'occhio risulta ben corretto dall'aberrazione sferica per tutte le condizioni di illuminazione diurna, dall'alba al tramonto (pupilla $\leq 4$ $\mathrm{mm}$ ), garantendo una prestazione quasi perfetta (diffraction limited). In questo intervallo di luminanza, l'aberrazione cromatica, indubbiamente legata ad una difficoltà progettuale relativa ai materiali delle ottiche rifrattive, rimane contenuta entro livelli modesti. Il progressivo deterioramento di prestazione al diminuire della luminosità è funzionale all'esigenza di disporre di un'elevata AV esclusivamente nel corso del dì, lasciando per i momenti di penombra una visione più modesta e per il buio un semplice sistema visivo di allerta e non di riconoscimento vero e proprio.

In conclusione, l'occhio appare come il punto di arrivo di un sofisticatissimo processo evolutivo volto a ottimizzare le prestazioni finalizzate a precise attività in condizioni ben definite. In questo senso non è sostenibile la tesi di Navarro per cui l'evoluzione avrebbe perso l'opportunità di fornire all'uomo una qualità visiva molto migliore. No, l'evoluzione ha prodotto il sistema visivo più utile all'uomo nel contesto in cui egli e i suoi antenati si sono evoluti in milioni di anni. Basti pensare allo sforzo non banale operato per correggere l'aberrazione sferica con superfici asferiche e un cristallino a gradiente d'indice: davvero un'autentica meraviglia della natura, anche se l'effetto è limitato all'ambito della visione fotopica. I difetti della visione umana risultano riscontrabili soprattutto in condizioni di ridotta illuminazione: evidentemente l'evoluzione non si è ancora accorta che da qualche millennio l'uomo dispone di illuminazione artificiale per interni! (Per contro noi abbiamo accelerato l'evoluzione con l'adozione di abitudini dannose quale l'iperstimolazione dello sforzo accomodativo fin dalla tenera età, che ha portato alla proliferazione della miopia nella popolazione scolarizzata, sviluppando una specie di "homo miopicus"). La visione umana dunque non è stata progettata per la discriminazione di stelle doppie o per la lettura in penombra di pagine stampate, ma per agevolare l'esecuzione di tutte le attività più necessarie alla sopravvivenza quotidiana: caccia, raccolta, riconoscimento di amici e nemici, interpretazione di espressioni, individuazione di pericoli, ecc. A tal fine il sistema visivo non è un semplice registratore di immagini della realtà circostante (come la videocamera di una Google car), ma uno strumento di interpretazione e predizione dell'ambiente secondo canoni predefiniti; lo dimostra la pervicace con- 
vinzione di vedere triangoli nel disegno di Fig. 5, laddove non ne è presente nemmeno uno. Forse anche questo da annoverare tra $\mathrm{i}$ "difetti" dell'occhio?

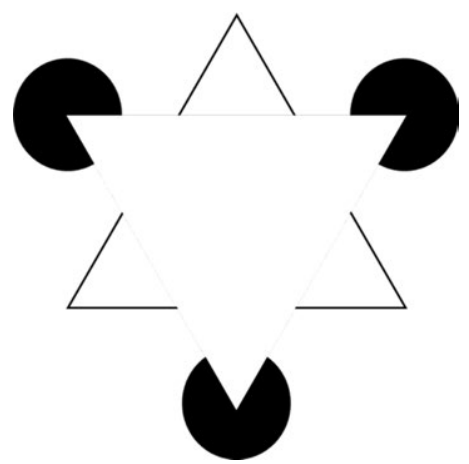

Fig. 5 - Illusione ottica: quanti sono $i$ triangoli, 1, 2, 8,..?

\section{BIBLIOGRAFIA}

1. Navarro, R. The Optical Design of the Human Eye: a Critical Review. J Optom, 2009: 2: 3-18.

2. Navarro, R. Aberrations of the Eye - Crude Flaws or Ecological Design? Reply to P.B. Kruger, J Optom, 2009: 2: 163-164.

3. Gobbi, PG. Modeling the Optical and Visual Performance of the Human Eye. SPIE Press, Bellingham, 2013.

4. Gullstrand, A. The optical system of the eye. In: Helmboltz's Treatise on Physiological Optics, Vol. I, Appendix 11.3. Southall, JPC, ed., The Optical Society of America, 1924.

5. Campbell, FW, Gubisch, RW. Optical Quality of the Human Eye. J Physiol, 1966: 186: 558-578.

6. Barten, PGJ. Contrast Sensitivity of the Human Eye and Its Effects on Image Quality. SPIE Optical Engineering Press, Bellingham, 1999.

7. Elliott, DB, Yang, KCH, Whitaker, D. Visual Acuity Changes Throughout Adulthood in Normal, Healthy Eyes: Seeing Beyond 6/6. Optom Vis Sci, 1995: 72: 186-191.

8. Hubel, DH, Wiesel, TN. Receptive fields, binocular interaction and functional architecture in the cat's visual cortex. J Physiol, 1962: 160: 106-154.

9. Snowden, R, Thompson, P, Troscianko, T. Basic Vision: An introduction to visual perception. Oxford University Press, Oxford, 2006. 